\section{ACOUSTICS, LIGHT, AND HEAT}

Acoustics, Light, and Heat. By William Lees, M.A., Lecturer on Natural Philosophy, the Heriot Watt College, and Lecturer on Mathematics and Experimental Physics, Free Church Training College, Edinburgh. New and Enlarged Edition. (London and Glasgow: Wm. Collins, Sons, and Co.)

THIS is one out of many of the text-books which have been called into existence by the "May" Examinations of the Science and Art Department. Being written especially to meet the requirements of the student who wishes to pass these examinations, it is only brought up to the standard given in the directory of the Department, and may therefore for this purpose be useful. The fact that a new and enlarged edition is now appearing is certainly evidence that this is the case. To make it more serviceable, the questions of all the May Examinations in Subject VIII. from 1872 to 1885 are given.

Though the simple and numerous diagrams and the generally clear nature of the text give it a certain value as a text-book, it is by no means so free from faults and ambiguities as might be expected in a new edition.

It may be well to refer especially to a few places where alterations suggest themselves.

Figs. 19 and 20 show the contrast between a musical sound and a noise. Though it is explained that "noises are due to irregular vibration or a confused mixture of musical sounds which produce aërial waves of great complexity and wanting in periodicity," no explanation is offered of a peculiarity in the "curve of a noise" (Fig. 20), which in three places is actually made to slope backwards.

Some of the figures in optics are rather wanting in precision. Thus in Fig. 73, which shows a real image formed by a concave mirror, a pair of slightly diverging rays are made to cross between the mirror and the principal focus. Again, Fig. 77 shows a caustic on the surface of milk in a glass with its cusp reaching close to the centre: Fig. I03 shows the action of a refracting plate on a beam of light by the turning and approximation of successive wave-fronts. Those two wave-fronts which obliquely cut the surface are shown straight and partly swung round, as if they were rigid lines meeting with resistance at one end. It would surely have been better to have bent the line at the point of intersection, leaving all the wave-fronts and parts of a front outside the medium parallel to one another, and also all inside parallel to one another, but it is possible that wave-fronts, strictly speaking, are not intended.

The explanation of so important a thing as the achromatic lens can hardly be considered satisfactory. Owing to its brevity it is possible to give this in full. "This defect in a lens [the defect of chromatic aberration] is obviated by the combination of a double convex lens of crown glass, with a convexo-concave of fint glass (Fig. I22). The effect of the second lens is to re-blend the coloured rays which the first has produced, and at the same time such an amount of refraction is preserved as to bring the light to a focus." As nowhere is it directly pointed out that for the same degree of refraction fint glass produces more dispersion than crown, it is not difficult to imagine that a student might fail to form any very clear idea of the principle of the achromatic lens, nor is he likely to be materially helped by the figure (122), which certainly does not represent either the section or any other view of any achromatic lens that was ever made. If it were not for the section lines it would be a good perspective drawing of a short cylinder; the ellipse which appears to be the end of such a cylinder is really meant to show the crown lens in section, and the figure of uniform thickness by its side, as thick everywhere as the ellipse is in the middle, which seems to be the side of the cylinder, is meant for the section of the flint lens. Simplicity in a diagram is a thing to be desired, but there is more than simplicity here.

Very little is said about spectrum analysis; and its application to the measurement of the motion of the heavenly bodies in the line of sight is not even mentioned.

The general weakness of the optical part is to a certain extent compensated for by the chapters on polarisation, which have much to recommend them. There is here, however, a paragraph which requires explanation. "Now it is found that whatever quantity of polarised light there is for any incidence other than the polarising angle in the reflected beam, there is always the same quantity in the refracted beam. At the polarising angle, however, the refracted beam exhibits traces of polarisation." What is meant by this distinction is by no means clear.

Heat is more precisely and clearly treated than light, but here the general excellence is marred by an example to illustrate expansion in which the working out of the result shows that the obvious meaning of the question is not intended. What any one would understand by the words, "Find the length of a rod of brass which would expand equally with a rod of steel 3 feet long under a change of temperature of $10^{\circ} \mathrm{C}$.," is evidently -Find what length of brass will increase in length by the same amount that a 3 -foot rod of steel does for a change of $10^{\circ} \mathrm{C}$. But what is found in the working of the answer is the length of a piece of brass which will expand so as to be as long as a piece of steel 3 feet long when each is raised $10^{\circ} \mathrm{C}$.

Sufficient has been said to show that this book is not as clear and accurate in either the text or the figures as might be expected in a new edition.

\section{OUR BOOK SHELF}

Cholera Curable. By John Chapman, M.D. (London : Churchill, I 885.)

DR. ChAPMAN has had the opportunity of testing, in the Hôpital de la Charité in Paris, his method of the socalled neuro-dynamic treatment in Asiatic cholera, and his demonstration of the success of this treatment constitutes, we take it, the cardinal motive for the production of this book, although a good many other, mostly theoretical, considerations are brought into the discussion.

The symptoms of Asiatic cholera are explained by a number of assumptions on the action of the spinal cord and the sympathetic nervous system, but as to which we look in vain for experimental proof. The theories concerning the etiology and causation of cholera are fully treated, and then Dr. Chapman promises to furnish us with a complete solution of these problems in a discovery made by him as to the cause of cholera. When, however, we come to analyse what he really has discovered, 